\title{
Minimum energy pathways via quantum Monte Carlo
}

\author{
S. Saccani, ${ }^{1}$ C. Filippi, ${ }^{2}$ and S. Moroni ${ }^{1}$ \\ ${ }^{1}$ SISSA Scuola Internazionale Superiore di Studi Avanzati and DEMOCRITOS National Simulation Center, \\ Istituto Officina dei Materiali del CNR Via Bonomea 265, I-34136, Trieste, Italy \\ ${ }^{2}$ MESA+ Institute for Nanotechnology, University of Twente, P.O. Box 217, \\ 7500 AE Enschede, The Netherlands
}

(Received 12 December 2012; accepted 6 February 2013; published online 28 February 2013)

\begin{abstract}
We perform quantum Monte Carlo (QMC) calculations to determine minimum energy pathways of simple chemical reactions, and compare the computed geometries and reaction barriers with those obtained with density functional theory (DFT) and quantum chemistry methods. We find that QMC performs in general significantly better than DFT, being also able to treat cases in which DFT is inaccurate or even unable to locate the transition state. Since the wave function form employed here is particularly simple and can be transferred to larger systems, we suggest that a QMC approach is both viable and useful for reactions difficult to address by DFT and system sizes too large for high level quantum chemistry methods. (C) 2013 American Institute of Physics. [http://dx.doi.org/10.1063/1.4792717]
\end{abstract}

\section{INTRODUCTION}

Determining minimum energy pathways of reactions is of fundamental importance in scientific and technological applications. The knowledge of barrier heights is a key to the prediction of catalytic properties of materials since it enables the use of transition state theory (TST) to determine reaction rates. ${ }^{1-3}$ Locating efficiently the transition state on a potential energy surface (PES) is in fact a popular subject in computational physics and, to this aim, a variety of algorithms have been developed such as the shallowest ascent, synchronous transit, and nudged elastic band (NEB) approaches. ${ }^{4}$ All these techniques ultimately rely on a method to determine the energy of a given atomic configuration and/or its derivatives.

If we restrict ourself to quantum simulations, the most used approaches are density functional theory (DFT) or highly-correlated quantum chemical methods, that is, wave function post-Hartree-Fock techniques such as the coupled cluster single-double and perturbative triple approach $[\operatorname{CCSD}(\mathrm{T})]$, which is generally considered the "gold standard" in quantum chemistry. Many of these wave function methods are variational (though coupled cluster methods are not) and in principle offer a systematic route to converge toward the exact energy, even though the increasing computational cost and the slow convergence severely limits this possibility. Their main drawback is that all these approaches implicitly or explicitly rely on expanding the wave function in Slater determinants and, therefore, require large amount of computer memory and have a poor size scaling ( $N^{7}$ for $\operatorname{CCSD(T),~} N$ being the number of electrons), limiting their range of applicability to small systems.

Consequently, for larger systems, DFT remains the method of choice due to its much more favorable computational cost (scaling from $N^{2}$ to $N^{4}$ ). Even though continuous progress in the field has led to the development of more precise and sophisticated DFT functionals, the situation is still far from satisfactory if one aims at high accuracy. ${ }^{5,6}$ For example, it is well known that popular functionals such as B3LYP $^{7-9}$ often lead to poor transition state geometries and barrier heights. ${ }^{10,11}$ Since DFT methods are not variational and do not offer a systematic way to improve their estimates, one has to resort to different approaches if better accuracy is needed.

Alternatively, one can employ quantum Monte Carlo (QMC) methods, such as variational (VMC) and diffusion (DMC) Monte Carlo. These well-established ab initio techniques take advantage of Monte Carlo integration over the full Hilbert space. In particular, VMC is a stochastic way of calculating expectation values of a complex trial wave function, which can be variationally optimized. DMC provides instead, with a higher computational cost, a stochastic groundstate solution to the full Schrödinger equation, given a fixed nodal surface (using the fixed-node approximation in order to avoid the notorious fermion sign problem). Because integrations are performed in the full Hilbert space, one can make use of non separable wave functions, with the explicit electron-electron correlation encoded in a so-called Jastrow factor. This allows for noteworthy accuracy already using a simple and non memory-intensive single determinant SlaterJastrow wave function. Although considerably more expensive than DFT methods (scaling as $N^{3}$ with a much larger prefactor), DMC generally offers better accuracy with respect to DFT. Furthermore, QMC methods possess a variational principle, which is a useful feature when one has to evaluate energy differences as in TST. From a computational point of view, QMC codes can be made to scale linearly with the number of cores and are not particularly memory demanding, making them suitable for today's massively parallel supercomputers. Finally, QMC methods offer in principle the possibility to push the calculation up to a desired accuracy by employing wave functions of increasing complexity (although, from a practical point of view, one is likely to adopt simple wave functions for intermediate-to-large sized systems 
due to the increased computational cost of multi-determinant wave functions).

In previous QMC studies of reaction barriers the geometries have been taken either from DFT, or from constrained geometry optimization along an assumed reaction coordinate. ${ }^{12-14}$ In this paper, we present nudged elastic band and climbing image calculations, ${ }^{15,16}$ where the geometry optimization of all the NEB images is done fully at the QMC level. For some representative challenging reactions from the NHTBH38/04 database $\mathrm{e}^{10,17}$ and for a hydrogen transfer reaction, ${ }^{18}$ we determine transition state geometries and forward-reverse barrier heights within VMC and DMC, and compare our results against several current DFT functionals and other wave function methods. We demonstrate that VMC is able to locate reaction geometries with higher accuracy than DFT, while DMC outperforms DFT in evaluating barrier heights. Thus a sensible strategy, in terms of accuracy versus computational cost, is to calculate DMC barrier heights on VMC geometries.

\section{METHODOLOGY}

The computation of the reactant and product geometries, the NEB calculations, and the saddle-point location through the climbing-image method are all optimization procedures over the total energy, although with different constraints. In particular, we use here the Newton optimization method, based on the knowledge of the first and second derivatives of the energy. For the reactions reported here, these derivatives are from QMC calculations based on correlated sampling but, for larger systems, analytic calculation of QMC forces is possible and advisable in order to reduce computational effort. ${ }^{19}$ Unless otherwise stated, we employ a single determinant Slater-Jastrow wave function:

$$
\Psi(r, R)=D^{\uparrow}\left(\phi^{\uparrow}, r^{\uparrow}, R\right) D^{\downarrow}\left(\phi^{\downarrow}, r^{\downarrow}, R\right) J(r, R),
$$

where $\{r\}$ and $\{R\}$ denote the electronic and nuclear positions, respectively. The Jastrow factor, $J(r, R)$, explicitly depends on the inter-particle coordinates, and includes electronelectron, electron-nucleus, and electron-electron-nucleus correlation terms..$^{20}$ The Slater determinants, $D^{\uparrow}$ and $D^{\downarrow}$, are constructed from the sets of molecular orbitals $\left\{\phi^{\uparrow}\right\}$ and $\left\{\phi^{\downarrow}\right\}$ for the up- and down-spin electrons, respectively. We employ scalar-relativistic energy-consistent Hartree-Fock pseudopotentials specifically constructed for QMC calculations and expand the molecular orbitals on the corresponding ccpVDZ basis set. ${ }^{21-23}$ These pseudopotentials have been extensively benchmarked, and their reliability has been recently supported by a DMC computation of atomization energies to near-chemical accuracy. ${ }^{24}$ The pseudopotentials are treated beyond the locality approximation. ${ }^{25}$

Our choice of such minimal wave function and basis set is intentional since we want to maximize the scalability of our approach to systems larger than the ones considered here. Our interest here is not to challenge quantum chemistry methods for small systems but, rather, to devise a strategy that has a more extended range of applicability while preserving a notable accuracy. While most DMC calculations found in literature use molecular orbitals computed with some other elec- tronic structure method, ${ }^{26-32}$ most often DFT, a key feature of our approach is that it is fully consistent since, at each iteration step in our geometric optimization, we perform a QMC optimization of all wave function parameters. This is done in order to guarantee consistency between the forces and the PES (see below) as well as to improve the results in terms of the absolute energy. The wave function optimization is performed at VMC level and details of the procedure are described elsewhere. ${ }^{33}$ It is found that this optimization procedure only approximately doubles the computer time needed to perform the calculations, while significantly lowering the expectation value of the energy.

The QMC calculations are performed with a modified version of the CHAMP program. ${ }^{34}$ Since forces calculated in QMC possess a statistical uncertainty, strictly speaking the optimization procedure via the Newton method never converges. Therefore, the equilibrium positions of the images along the nudged elastic band are obtained by averaging over several iterations after all quantities vary only by statistical fluctuations around a stationary value. We use a time step of 0.01 a.u. in the DMC calculations. The DFT and HartreeFock (HF) all-electron calculations are performed with the GAMESS package, ${ }^{35}$ using Dunning-type Correlation Consistent triple-zeta basis sets, augmented with a set of diffuse function (aug-cc-pVTZ). The QMC calculations employ instead scalar-relativistic pseudopotentials. For the light elements considered here, these relativistic effects are small and will not affect our results.

\section{RESULTS}

We select four challenging reactions from the NHTBH38/04 database ${ }^{10}$ plus one hydrogen transfer reaction. As best estimates, we use the atomic geometries for the initial, final, and transition states reported in the database and computed through a quadratic configuration interaction with single and double excitations (QCISD) optimization. For these geometries, the barrier heights estimated with the W1 method (a complete basis set extrapolation over $\operatorname{CCSD}(\mathrm{T})$ ) are also available. The reference data for $\mathrm{H}+\mathrm{OH} \rightarrow \mathrm{H}_{2}+\mathrm{O}$ are from ext-CAS $+1+2+\mathrm{Q}$ calculations. ${ }^{18}$

We initially focus on the $\mathrm{H}+\mathrm{F}_{2} \rightarrow \mathrm{HF}+\mathrm{F}$ reaction and collect the DFT and QMC data in Table I. To measure how much the geometries differ from the best estimates, we calculate the RMS deviations of the interatomic distance among all atoms in the initial/final/transition state configurations with respect to the corresponding best-estimate geometries. In the table, a forward barrier $\left(V_{f}\right)$ of zero means that the DFT functional finds no transition state (i.e., the reactants are unstable) with the reverse barrier being the reactant-product energy difference. Most DFT functionals fail in finding any transition state for this reaction, including the hybrid functionals

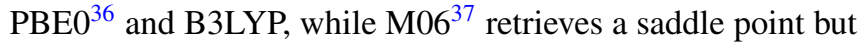
with large deviations over the best estimate transition state geometry. In Table I, we also report the initial/final/transition state geometries computed via VMC forces, where the uncertainty on the interatomic bonds due to the statistical noise on the forces is about $0.002 \AA$. These geometries come from a fully VMC NEB and climbing image calculations. VMC is 
TABLE I. Barrier heights and RMS of geometric deviations, $\mathrm{H}+\mathrm{F}_{2} \rightarrow \mathrm{HF}+\mathrm{F}$ reaction, for reactants (React), products (Prod) and transition states (TS). We denote with $V_{f} / V_{r}$ the forward/reverse reaction barrier heights (BHs). The RMS is calculated over the deviation of the interatomic distances of all the atoms from the best estimated geometry.

\begin{tabular}{|c|c|c|c|c|c|c|c|c|c|c|c|c|c|}
\hline $\mathrm{H}+\mathrm{F}_{2} \rightarrow \mathrm{HF}+\mathrm{F}$ & & $\mathrm{BE}$ & VMC & VMC CAS & $\mathrm{DMC}$ & DMC CAS & $\mathrm{HF}$ & LSDA & BLYP & B3LYP & PBE & PBE0 & M06 \\
\hline \multirow[t]{2}{*}{$\mathrm{BHs}(\mathrm{Kcal} / \mathrm{mol})$} & $V_{f}$ & 2.27 & $6 \pm 1$ & $1.3 \pm 0.2$ & $2.2 \pm 0.5$ & $1.4 \pm 0.3$ & 0.0 & 0.0 & 0.0 & 0.0 & 0.0 & 0.0 & 2.7 \\
\hline & $V_{r}$ & 106.18 & $126 \pm 1$ & $112.2 \pm 0.6$ & $114 \pm 1$ & $105.4 \pm 0.7$ & 127.3 & 83.2 & 90.9 & 100.9 & 87.9 & 100.0 & 107.8 \\
\hline \multirow[t]{3}{*}{ RMS deviation $(\AA)$} & React & & 0.008 & & 0.007 & & 0.067 & 0.010 & 0.037 & 0.002 & 0.018 & 0.019 & 0.020 \\
\hline & Prod & & 0.002 & & 0.008 & & 0.016 & 0.018 & 0.020 & 0.009 & 0.017 & 0.004 & 0.001 \\
\hline & $\mathrm{TS}$ & & 0.028 & & 0.013 & & $\ldots$ & $\ldots$ & $\ldots$ & $\ldots$ & $\ldots$ & $\ldots$ & 0.216 \\
\hline
\end{tabular}

able to retrieve even at the single-determinant level the initial, the final and, especially, the transition state geometry with much better accuracy than DFT. It is interesting to notice that, for geometrical data, VMC also performs better than the hybrid-meta M06 functional which is constructed to fit the barrier heights calculated on the best-estimate geometries from the database. ${ }^{37}$ Clearly, this fitting procedure does not always guarantee that the actual transition state retrieved by the functional is near the best-estimate one. For testing purposes we also have located the saddle point using non optimized orbitals from the M06 functional and a fixed Jastrow; we find that the result is substantially worse, with a RMS deviation from the best estimate of $0.149 \AA$. This result confirms that at least in some cases the wave function optimization is needed to obtain accurate values.

Although the VMC geometries are rather accurate, the predicted reverse energy barrier $\left(V_{r}\right)$ is markedly overestimated. Performing a DMC calculation on the VMC geometries retrieves better energy estimates, but the error on the reverse barrier of about $8 \mathrm{Kcal} / \mathrm{mol}$ is still quite significant. We find that, to improve this energy barrier, it is not useful to reoptimize the geometries via DMC forces: the use of DMC forces does not alter significantly the geometries (within a statistical uncertainty on the transition state geometry of about $0.008 \AA$ ) and, consequently, the estimated barrier heights either. In order to improve over these values, it is possible to take advantage of the variational principle available in QMC and to resort to the use of multi-determinantal wave function. In this case, we employ a simple complete active space (CAS) wave function correlating three electrons in the three active orbitals relevant for the reaction and recompute the energy barriers over the VMC geometries obtained at the single-determinant level. The resulting VMC barrier heights are improved and the DMC values become very similar to the best estimates. Although the use of CAS wave functions is not readily applicable to larger systems due to their exponential scaling with system size, there exists scalable techniques to improve over singledeterminant wave functions through the design of accurate multi-determinantal size-extensive and linear-scaling ${ }^{38}$ or backflow wave functions. ${ }^{39,40}$

We now return to the simple Slater-Jastrow wave function, and consider the other reactions. In Figure 1, we compare the difference between the geometries obtained by VMC and various DFT functionals with respect to the reference ones obtained with QCISD, using the same measuring criterion as in Table I. The functionals reported in this figure are all hybrid or meta-hybrid and are the ones returning the smallest geometric/energy deviation among the ones we tested on this set of reactions, which are the same ones listed in Table I. The generalized-gradient-approximation functionals used in the overwhelming majority of DFT applications of CI-NEB, entail significantly larger deviations. For example, the deviation of PBE is two to four times larger than that of PBE0 for transition state geometries reported here, and the RMS barrier heights deviation of PBE on this set is more than twice than that of PBE0. For equilibrium geometries, VMC performs at the level of the hybrid functionals. For the transition state, it typically returns more accurate geometries, often performing much better than DFTs. Notwithstanding that the M06 is actually fitted to reproduce barrier heights for the NHTBH38/04 reactions, VMC still performs better than this functional in evaluating transition state geometries. This may be again due to the M06 parametrization procedure, which does not
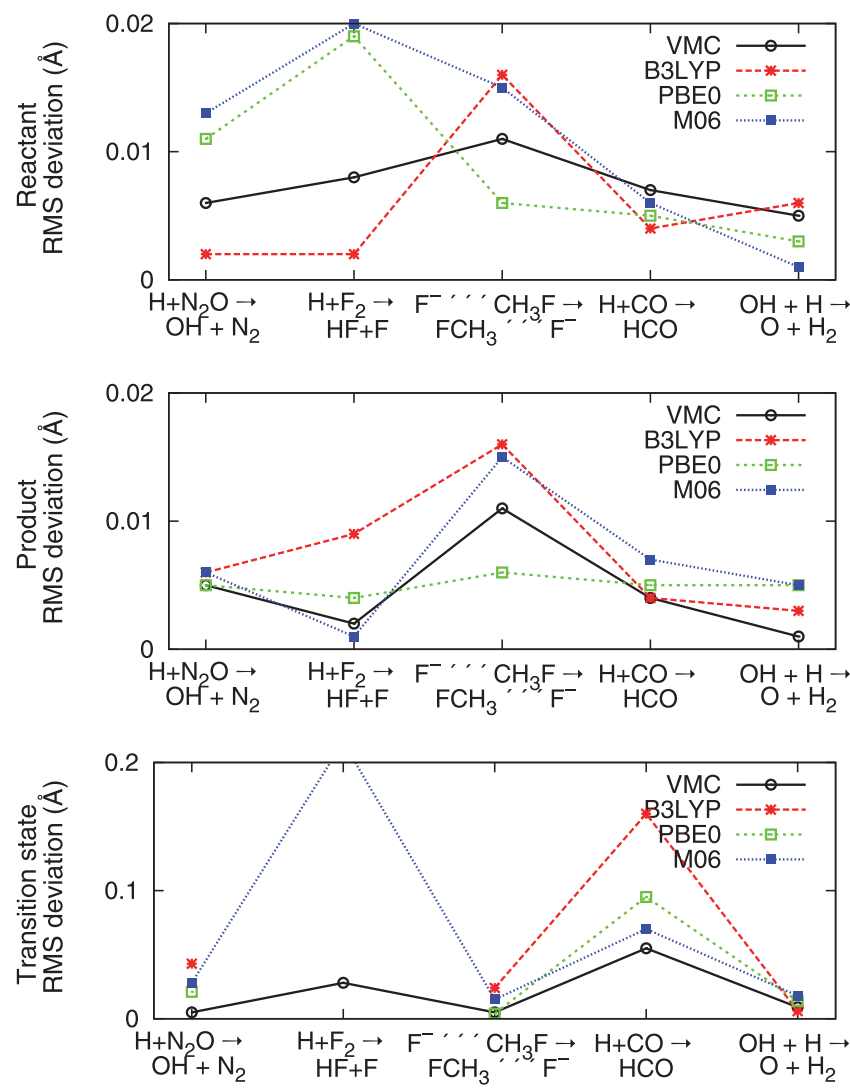

FIG. 1. RMS of deviation of interatomic distances from the QCISD geometries $(\AA)$. Distances are calculated among all atoms involved in the reactions. 

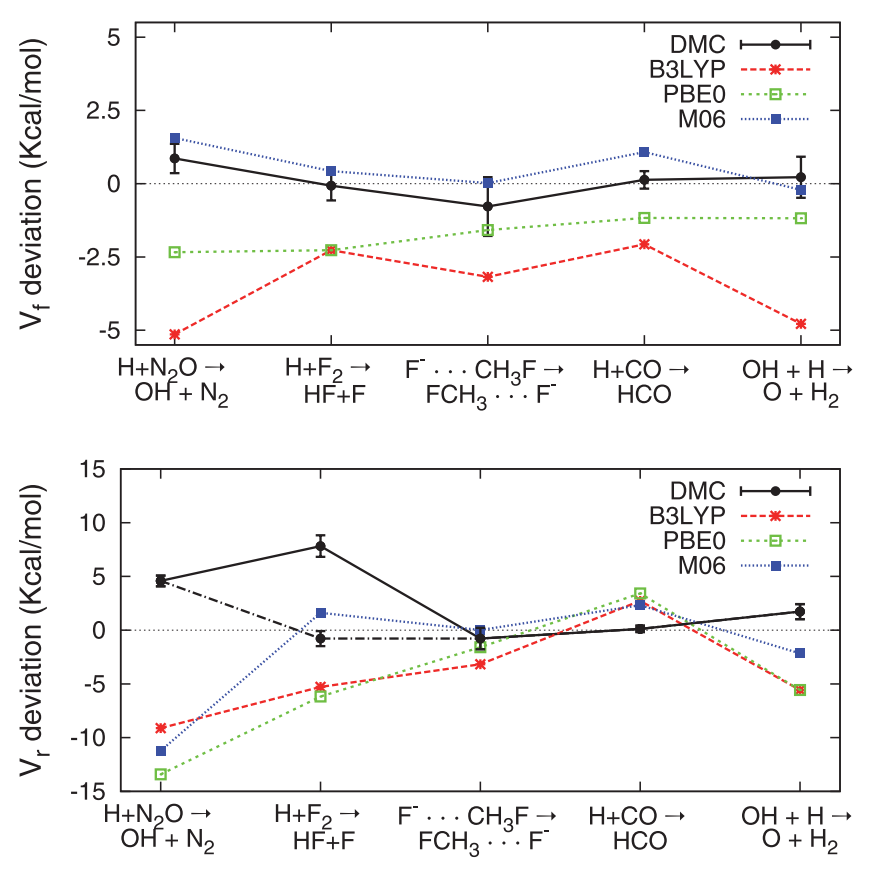

FIG. 2. Forward $\left(V_{f}\right)$ and reverse $\left(V_{r}\right)$ barrier heights deviation from best estimates calculated by QMC and DFT methods (Kcal/mol). The dasheddotted line represents the DMC values obtained by employing the CAS wave function for the $\mathrm{H}+\mathrm{F}_{2} \rightarrow \mathrm{HF}+\mathrm{F}$ instead of the single determinant one (full line), see text.

guarantee the accuracy of the saddle point located by the functional. We do not recalculate the geometries employing DMC forces because, from the test performed, DMC forces improves VMC transition state geometries only slightly, as these are already notably accurate. Furthermore, DMC geometry corrections are barely reflected in the calculation of the barrier heights, as these energies are second order in the deviation from the actual equilibrium points. For these reasons, we speculate that the calculations of geometries at VMC level and of barrier heights at DMC level is the most sensible choice regarding the trade-off between accuracy and computational cost.

In Figure 2, we report the forward and reverse reaction barriers. While VMC (not shown) performs less accurately than hybrid functionals, DMC calculated on the VMC geometries significantly improves the barrier heights of all reactions upon the VMC values, and performs at the level of the hybrid DFT approaches. In particular, our QMC procedure is more accurate than the hybrid functionals B3LYP and PBE0, while the only functional performing on average at the same level or slightly better is M06, despite these barriers being calculated on transition state geometries worse than VMC ones. Moreover M06 is actually fitted to reproduce precisely the NHTBH38/04 barrier heights, and there is no guarantee that, on a different set of reactions, it would still perform as well as QMC. Note that single-determinant DMC performs better than all DFT approaches, included M06, even in the reaction $\mathrm{H}+\mathrm{N}_{2} \mathrm{O} \rightarrow \mathrm{OH}+\mathrm{N}_{2}$, which is known to be strongly multi-determinantal in character. Overall, QMC gets both the geometry and the energetics accurately, offering a parameter-free, more balanced description of reactants, products and transition states than all DFT schemes considered here.

\section{REMARKS ON QMC FORCES CALCULATION}

We compute here the VMC and DMC energy derivatives with correlated sampling. While the procedure is relatively straightforward in VMC, the calculation of forces in DMC is more involved and approximations are generally used. For details about the DMC algorithm, we refer the reader to Ref. 41. In both VMC and DMC, one should know the derivatives of the energy with respect to the wave function parameters and of the parameters with respect to the nuclear coordinates in order to calculate the forces correctly. In VMC, we avoid this problem by fully optimizing the wave function so that all the derivatives with respect to the parameters are zero. In DMC, the bias in the forces due to wave function optimization at VMC level has been found negligible in a few test cases.

\section{v. CONCLUSIONS}

We investigated the possibility of performing full-QMC reconstruction of minimum energy pathways of chemical reactions. Full optimization of the wave function parameters is carried out during each iteration, so the employed technique is internally fully consistent. Geometric optimization of the minimum energy pathway and of the transition state is done at $\mathrm{VMC}$ level, with the obtained geometries being more accurate than DFT ones, especially for transition states. It also demonstrates the ability to correctly locate the transition state in cases in which DFT fails in returning accurate geometries. At DMC level, the method displays very good performance in evaluating barrier reaction heights, comparing favorably even against hybrid functionals. Therefore, our approach of calculating the geometries at the VMC level and the barrier heights at the DMC level is most effective as far as performance over computational cost is concerned: calculating DMC geometries is very expensive and, in the tested cases, it does not improve significantly the estimates, while calculating DMC energies over VMC geometries is much cheaper and still retrieves good results. Since the employed wave function is of the simple Slater-Jastrow type, this technique is scalable to larger systems. Our results indicate that, for intermediatesized system reactions where quantum chemistry methods are not computationally viable, the QMC approach may be the most accurate technique currently available.

\section{ACKNOWLEDGMENTS}

We acknowledge Dr. O. Valsson for assistance in the usage of CHAMP and GAMESS packages, and Professor S. Baroni for helpful discussions.

\footnotetext{
${ }^{1}$ H. Eyring, J. Chem. Phys. 3, 107 (1935).

${ }^{2}$ E. Wigner, Trans. Faraday Soc. 34, 29 (1938).

${ }^{3}$ J. C. Keck, Adv. Chem. Phys. 13, 85 (1967).

${ }^{4}$ C. Dykstra, G. Frenking, K. Kim, and G. Scuseria, Theory and Applications of Computational Chemistry: The First Forty Year (Elsevier, 2005), Chap. 10.

${ }^{5}$ Y. Zhang and W. Yang, J. Chem. Phys. 109, 2604 (1998).

${ }^{6}$ D. R. B. Brittain, C. Y. Lin, A. T. B. Gilbert, E. I. Izgorodina, P. M. W. Gill, and C. L. Michelle, Phys. Chem. Chem. Phys. 11, 1138 (2009).

${ }^{7}$ C. Lee, W. Yang, and R. G. Parr, Phys. Rev. B 37, 785 (1988).
} 
${ }^{8}$ A. D. Becke, J. Chem. Phys. 98, 5648 (1993).

${ }^{9}$ P. J. Stephens, F. J. Devlin, C. F. Chabalowski, and M. Frisch, J. Phys. Chem. 98, 11623 (1994).

${ }^{10}$ Y. Zhao, N. Gonzlez-Garca, and D. G. Truhlar, J. Phys. Chem. A 109, 2012 (2005).

${ }^{11}$ M. T. Nguyen, S. Creve, and L. G. Vanquickenborne, J. Phys. Chem. 100, 18422 (1996).

${ }^{12}$ J. C. Grossman and L. Mitas, Phys. Rev. Lett. 79, 4353 (1997).

${ }^{13}$ M. Barborini and L. Guidoni, J. Chem. Phys. 137, 224309 (2012).

${ }^{14}$ C. Filippi, S. Healy, P. Kratzer, E. Pehlke, and M. Scheffler, Phys. Rev. Lett. 89, 166102 (2002).

${ }^{15}$ G. Mills and H. Jonsson, Phys. Rev. Lett. 72, 1124 (1994).

${ }^{16}$ G. Helkelman, B. P. Uberuaga, and H. Jónsson, J. Phys. Chem. 113, 9901 (2000).

${ }^{17}$ Y. Zhao, B. J. Lynch, and D. G. Truhlar, Phys. Chem. Chem. Phys. 7, 43 (2005).

${ }^{18}$ K. A. Peterson and T. H. Dunning, J. Phys. Chem. A 101, 6280 (1997).

${ }^{19}$ S. Sorella and L. Capriotti, J. Chem. Phys. 133, 234111 (2010).

${ }^{20}$ C. Filippi and C. J. Umrigar, J. Chem. Phys. 105, 213 (1996). As Jastrow correlation factor, we use the exponential of the sum of three fifthorder polynomials of the electron-nuclear, the electron-electron, and of pure 3-body mixed e-e and e-n distances, respectively. The Jastrow factor is adapted to deal with pseudo-atoms, and the scaling factor $\kappa$ is set to 0.60 a.u.

${ }^{21}$ M. Burkatzki, C. Filippi, and M. Dolg, J. Chem. Phys. 126, 234105 (2007).

${ }^{22}$ M. Burkatzki, C. Filippi, and M. Dolg, J. Chem. Phys. 129, 164115 (2008).

${ }^{23}$ For the hydrogen atom, we use a more accurate BFD pseudopotential and basis set. M. Dolg and C. Filippi, private communication, 2012.

${ }^{24}$ F. R. Petruzielo, J. Toulouse, and C. J. Umrigar, J. Chem. Phys. 136, 124116 (2012).
${ }^{25}$ M. Casula, Phys. Rev. B 74, 161102(R) (2006).

${ }^{26}$ A. J. Williamson, J. C. Grossman, R. Q. Hood, A. Puzder, and G. Galli, Phys. Rev. Lett. 89, 196803 (2002).

${ }^{27}$ L. K. Wagner and L. Mitas, Chem. Phys. Lett. 370, 412 (2003).

${ }^{28}$ E. R. Batista, J. Heyd, R. G. Hennig, B. P. Uberuaga, R. L. Martin, G. E. Scuseria, C. J. Umrigar, and J. W. Wilkins, Phys. Rev. B 74, 121102(R) (2006).

${ }^{29}$ E. Sola, J. P. Brodholt, and D. Alfé, Phys. Rev. B 79, 024107 (2009).

${ }^{30}$ L. K. Wagner and J. C. Grossman, Phys. Rev. Lett. 104, 210201 (2010).

${ }^{31}$ K. P. Driver, R. E. Cohen, Z. Wu, B. Militzer, P. Lpez Ros, M. D. Towler, R. J. Needs, and J. W. Wilkins, Proc. Natl. Acad. Sci. U.S.A. 107, 9519 (2010).

${ }^{32}$ R. Q. Hood, P. R. C. Kent, and F. A. Reboredo, Phys. Rev. B 85, 134109 (2012).

${ }^{33}$ C. J. Umrigar, J. Toulouse, C. Filippi, S. Sorella, and R. G. Hennig, Phys. Rev. Lett. 98, 110201 (2007).

${ }^{34}$ CHAMP is a quantum Monte Carlo program package written by C. J. Umrigar, C. Filippi, and collaborators.

${ }^{35}$ M. W. Schmidt et al., J. Comput. Chem. 14, 1347-1363 (1993).

${ }^{36}$ C. Adamo and V. Barone, J. Chem. Phys. 110, 6158 (1999).

${ }^{37}$ Y. Zhao and D. G. Truhlar, Theor. Chem. Acc. 120, 215 (2008).

${ }^{38}$ F. Fracchia, C. Filippi, and C. Amovilli, J. Chem. Theory Comput. 8, 1943 (2012).

${ }^{39}$ M. Holzmann, D. M. Ceperley, C. Pierleoni, and K. Esler, Phys. Rev. E 68, 046707 (2003); M. Holzmann, B. Bernu, and D. M. Ceperley, Phys. Rev. B 74, 104510 (2006).

${ }^{40}$ P. Lopez Rios, A. Ma, N. D. Drummond, M. D. Towler, and R. J. Needs, Phys. Rev. E 74, 066701 (2006).

${ }^{41}$ C. Filippi and C. J. Umrigar, Phys. Rev. B 61, R16291 (2000). 